TRANSACTIONS OF THE

AMERICAN MATHEMATICAL SOCIETY

Volume 352, Number 8, Pages 3707-3721

S 0002-9947(00)02510-

Article electronically published on March 2, 2000

\title{
WEAK AND STRONG DENSITY OF COMPOSITIONS
}

\author{
LUIGI DE PASCALE AND EUGENE STEPANOV
}

Dedicated to N.V. Azbelev on the occasion of his 75th birthday

\begin{abstract}
The convergence in various topologies of sequences of inner superposition (composition) operators acting between Lebesgue spaces and of their linear combinations is studied. In particular, the sequential density results for the linear span of such operators is proved for the weak, weak continuous and strong operator topologies.
\end{abstract}

\section{INTRODUCTION}

Usually when dealing with homogenization or asymptotic analysis problems for differential equations, one is required to study various types of convergence of the operators involved. The situation becomes especially complicated, when the structure of the problem under consideration is essentially determined not only by local operators on function spaces (e.g., the differential operator in spaces of differentiable functions, Nemytskiǐ operator in Lebesgue spaces), but also by nonlocal ones.

Among linear nonlocal operators an important role is played by the inner superposition (sometimes also called composition or shift) operator, which naturally appears in functional differential equations with deviating argument and in the related variational problems. In the spaces of functions over $\Omega \subset \mathbb{R}^{n}$ it is defined formally by the relationship

$$
\left(T_{g} u\right)(x)=\left\{\begin{array}{cc}
u(g(x)), & g(x) \in \Omega, \\
0, & g(x) \notin \Omega,
\end{array}\right.
$$

where $g: \Omega \rightarrow \mathbb{R}^{n}$ is a given generating function. The operators of this type can often be considered the "elementary" carrier of nonlocality. In most applications to functional differential equations one has to study inner superposition operators defined over some space of summable functions, like Lebesgue or Orlicz space.

The study of strong convergence of inner superpositions in various ideal Banach spaces and in the space of measurable functions has been started by M.E. Drakhlin (see [9] and references therein) in order to enable the study of continuous dependence on parameters of solutions to FDE's. At that time necessary and sufficient conditions were found for a sequence of inner superpositions to converge to an operator of the same type. Recently, however, the applications to variational and optimal control problems related to FDE's made it important to study also weaker concepts of convergence of nonlocal operators, especially weak operator convergence and so-called continuous weak convergence (the latter in the reflexive Lebesgue

Received by the editors May 5, 1997 and, in revised form, March 11, 1998.

1991 Mathematics Subject Classification. Primary 47B38, 47A67, 34K05.

(C)2000 American Mathematical Society 
space framework is equivalent to the strong convergence of adjoints). As it has been shown in [7] 2], it is exactly the latter convergence that plays the crucial role in nonlocal homogenization and $\Gamma$-convergence problems. Moreover, such applications revealed the necessity to put stress on representation of the limit operator. In particular, from the results of [2] it follows that it is essentially the form of strong or continuous weak limits of inner superposition operators that determines the structure of the variational limits of optimal control problems for differential equations with deviating argument. The respective study of convergence of inner superpositions in weak operator topologies has been started in [11. In particular, it has been shown that a sequence of inner superpositions in a separable Lebesgue space converges weakly to an inner superposition operator, if and only if the convergence is strong. However, in 11] and in the successive paper 10 the examples have been provided to show that still inner superpositions can converge weakly (and even continuously weakly) to an operator of a different type, e.g. to a regular integral operator. This gives a hint that all the operators between appropriate Lebesgue spaces might be approximated by inner superposition operators in a sufficiently weak sense.

In the present paper we will follow this indication and show that the linear span of the class of all inner superpositions between appropriate Lebesgue spaces is sequentially dense in the respective space of linear operators in weak (and even continuous weak) operator topology. Proving this we are able to construct explicitly the sequence of linear combinations of inner superpositions approximating in the desired weak sense any given operator. The generating functions of the respective approximating operators are, in fact, rather "nice", being local homeomorphisms. Moreover, exploiting the results of A. Chistyakov [3], we show that it is possible to approximate any given operator between appropriate Lebesgue spaces by a sequence of linear combinations of inner superpositions converging both strongly and continuously weakly. The proof of the latter result, however, will not be constructive, and will involve generating functions which are very "irregular".

\section{NOTATION AND PRELIMINARIES}

In what follows we consider generically $\Omega \subset \mathbb{R}^{n}$ to be an open bounded set (extra regularity requirements will be added upon necessity). For a measurable set $e \subset \mathbb{R}^{n}$ we denote by $|e|$ its measure and by $\chi_{e}$ its characteristic function. $\chi_{\Omega}$ sometimes will also be denoted by $1_{\Omega}$. The Lebesgue spaces $L^{p}(\Omega)$ equipped with their usual norm $\|\cdot\|_{p}$ will be often abbreviated by $L^{p}$. We note that in principle most of the results claimed below remain valid with some obvious adjustments upon further generalizations (e.g., substituting $\Omega \subset \mathbb{R}^{n}$ with Lebesgue measure by a generic measure space with a finite nonatomic measure and Lebesgue spaces by "nice" ideal Banach spaces). Nevertheless, we prefer not to overload the paper with additional complications. For the norm of a linear operator acting between the spaces $L^{p}$ and $L^{q}$ we use the notation $\|\cdot\|_{p \rightarrow q}$.

Let us recall some known results from [1, 8] about inner superposition operators acting between Lebesgue spaces. The operator $T_{g}$ is well-defined by the formula (S), if and only if the generating function $g: \Omega \rightarrow \mathbb{R}^{n}$ is measurable and satisfies the additional requirement

$$
e \subset \Omega, \quad|e|=0 \Rightarrow \mu_{g}(e):=\left|g^{-1}(e)\right|=0,
$$


which we will always consider fulfilled in the sequel. This condition provides the existence of a Radon-Nikodym derivative $\frac{d \mu_{g}}{d m} \in L^{1}$ of the image measure $\mu_{g}$ with respect to the Lebesgue measure. The same condition is necessary and sufficient for acting and continuity of $T_{g}$ in the space $\mathcal{M}:=\mathcal{M}(\Omega)$ of (classes of) measurable functions with the topology of convergence in measure.

The operator $T_{g}$ acts between the spaces $L^{p}$ and $L^{q}$, if and only if $q \leq p$ and $\frac{d \mu_{g}}{d m} \in L^{p /(p-q)}$. The condition of continuity coincides with acting condition, since $T_{g}$ is positive (i.e. maps nonnegative functions in nonnegative ones) [14]. Note that any nonzero inner superposition operator acting between Lebesgue spaces is noncompact. At last, we $\operatorname{denote} \mathcal{E}(e):=g^{-1}(e)$ and $\mathcal{E}:=\mathcal{E}(\Omega)$.

\section{Abstract Results on operator CONVERgEnCE}

In this section unless otherwise explicitly mentioned, we always consider $A_{\nu}, A$ : $X \rightarrow Y$ to be linear operators between Banach spaces $X$ and $Y, X^{\prime}$ and $Y^{\prime}$ stand for the duals of $X$ and $Y$ respectively and denote by $A_{\nu}^{\prime}, A^{\prime}: Y^{\prime} \rightarrow X^{\prime}$ the adjoints of the operators $A_{\nu}, A: X \rightarrow Y$. The space of linear operators between $X$ and $Y$ will be denoted $\mathcal{L}(X, Y)$.

Definition 3.1. We say that the sequence $A_{\nu}$ converges to $A$

(U) uniformly, if it converges in the norm of $\mathcal{L}(X, Y)$;

(S) strongly, if $A_{\nu} x \rightarrow A x$ in $Y$ for all $x \in X$;

(W) weakly, if $A_{\nu} x \rightarrow A x$ in $Y$ for all $x \in X$;

(CW) continuously weakly, if $A_{\nu} x_{\nu} \rightarrow A x$ in $Y$ for any weakly converging sequence $x_{\nu} \rightarrow x$ in $X$.

For the weak and continuous weak convergence we adopt the notations $A_{\nu} \rightarrow A$ and $A_{\nu} \stackrel{(c w)}{\longrightarrow} A$ respectively. We remark that all the above types of convergence are induced by the respective topologies in $\mathcal{L}(X, Y)$, which we will refer to as uniform, strong, weak and continuous weak operator topologies respectively, of which in general only the uniform one is metrizable (see Chapter IV of [12]). In this paper however we operate in terms of sequences.

By $\left(\mathrm{U}^{\prime}\right),\left(\mathrm{S}^{\prime}\right)$ and $\left(\mathrm{W}^{\prime}\right)$ denote respectively the uniform, strong and weak (pointwise) convergences of the sequence $A_{\nu}^{\prime}$ to $A^{\prime}$. First we indicate the relationships between various types of operator convergence.

Lemma 3.1. The following implications hold:

$$
\begin{gathered}
\underset{\text { (S) }}{(\mathrm{U})} \\
(\mathrm{W}) \Leftrightarrow\left(\mathrm{W}^{\prime}\right) \Leftarrow(\mathrm{CW}) \Leftarrow \quad \begin{array}{r}
\left(\mathrm{S}^{\prime}\right) \\
\Uparrow \\
\left(\mathrm{U}^{\prime}\right)^{2} .
\end{array}
\end{gathered}
$$

Moreover, if $X$ is reflexive and $Y$ is uniformly convex (in particular, Hilbert), then $(\mathrm{CW}) \Leftrightarrow\left(\mathrm{S}^{\prime}\right)$. Any of the above types of convergence implies the uniform boundedness of the whole sequence and the boundedness of the limit operator.

For the proof of most of the implications the reader may consult [11; the others are trivial.

In the sequel the following result which deals with the "diagonal argument" in weak operator convergence will play a crucial role. 
Lemma 3.2. Let $X$ be reflexive and $Y$ be dual to some separable normed space (by weak convergence in $Y$ we understand then the *-weak one). Assume that $T_{\nu, \mu}, A_{\nu}$, $A \in \mathcal{L}(X, Y)$, while

$$
\begin{aligned}
& T_{\nu, \mu} \stackrel{(c w)}{\longrightarrow} \quad A_{\nu} \quad \text { as } \mu \rightarrow \infty \text { for each } \nu \in \mathbb{N} \text {, and } \\
& A_{\nu} \rightarrow A \quad \text { as } \nu \rightarrow \infty \text {. }
\end{aligned}
$$

Moreover, assume that

$$
\liminf _{\mu}\left\|T_{\nu, \mu}\right\|_{\mathcal{L}(X, Y)} \leq C
$$

for all $\nu \in \mathbb{N}$ and for some $C>0$ independent of $\nu$.

Then there exists a "diagonal" sequence $T_{\nu, \mu(\nu)}$ converging weakly to $A, T_{\nu, \mu(\nu)} \rightarrow$ A. Moreover, if the convergence of $A_{\nu}$ to $A$ is continuous weak, then also the mentioned diagonal sequence converges continuously weakly.

Proof. According to $(*)$, we may assume, up to extracting some subsequences in $\mu$, that the images of the unit ball in $X$ under the operators $T_{\nu, \mu}$, as well as under the operators $A_{\nu}$, and $A$ are all contained in some ball of $Y$. Since the *-weak topology in $Y$ is metrizable over any bounded set, denote by $d$ the respective metric inducing the $*$-weak topology in this ball of $Y$. First we prove that for any fixed $\nu \in \mathbb{N}$, there exists $\mu(\nu) \in \mathbb{N}$ such that

$$
d\left(T_{\nu, \mu(\nu)} x-A_{\nu} x, 0\right) \leq 1 / \nu \quad \forall x \in X:\|x\|=1 .
$$

In fact, supposing the contrary, we have that there are a $\nu \in \mathbb{N}$ and a sequence $\left\{x_{\mu}\right\}$ of elements of unit norm satisfying $d\left(T_{\nu, \mu} x_{\mu}-A_{\nu} x_{\mu}, 0\right) \geq 1 / \nu$. As the unit ball in $X$ is weakly compact, we may suppose $x_{\mu} \rightarrow x,\|x\| \leq 1$, and hence

$$
1 / \nu \leq d\left(T_{\nu, \mu} x_{\mu}-A_{\nu} x_{\mu}, 0\right) \rightarrow 0 \text { as } \mu \rightarrow \infty,
$$

leading to a contradiction. It remains now to observe that

$$
\begin{aligned}
d\left(T_{\nu, \mu(\nu)} x-A x, 0\right) & \leq d\left(T_{\nu, \mu(\nu)} x-A_{\nu} x, 0\right)+d\left(A_{\nu} x-A x, 0\right) \\
& \leq 1 / \nu+d\left(A_{\nu} x-A x, 0\right)
\end{aligned}
$$

while the right-hand side converges to zero for all $x \in X$ of unit norm, whence the weak convergence of the "diagonal" sequence follows. The same argument shows that the "diagonal" sequence converges continuously weakly when $A_{\nu} \stackrel{(c w)}{\longrightarrow} A$.

The following example shows that the requirement of weak continuous convergence of $T_{\nu, \mu}$ in the above assertion is essential and cannot be substituted by that of only weak convergence, even if $A_{\nu}$ are assumed to converge uniformly.

Example 3.1. Let $X=Y=L^{2}(0, \pi)$. Let $\left\{A_{\nu}\right\}$ be a constant sequence of operators, $A_{\nu}=0$, and define

$$
\left(T_{\nu, \mu} u\right)(x):=\nu \sin \mu x \cdot u(x) .
$$

Observe $T_{\nu, \mu} \rightarrow A_{\nu}=0$ for each $\nu \in \mathbb{N}$ as $\mu \rightarrow \infty$ weakly (but not weakly continuously) in $\mathcal{L}\left(L^{2}\right)$. Assume the existence of a "diagonal" subsequence $T_{\nu, \mu(\nu)} \rightarrow 0$. If $\mu(\nu)$ were bounded, then there would exist a $\bar{\mu} \in \mathbb{N}$ satisfying $\mu(\nu)=\bar{\mu}$ for infinitely many $\nu \in \mathbb{N}$. Therefore, taking $v(x):=\sin \bar{\mu} x$ and $u \equiv 1$, one would have 
$\left\langle T_{\nu, \mu(\nu)} u, v\right\rangle \rightarrow+\infty$ up to a subsequence, contradicting the assumption. Hence, we may suppose that up to a subsequence $\mu(\nu+1)>\mu(\nu)$. Now set

$$
v(x):=\sum_{k=1}^{\infty} \frac{1}{k} \sin \mu(k) x .
$$

Then $\left\langle T_{\nu, \mu(\nu)} u, v\right\rangle \nrightarrow 0$, for $u \equiv 1$, contradicting the assumption.

We remark also further the condition $(*)$ is necessary for the existence of a diagonal sequence as announced in the lemma.

\section{LIMITS OF INNER SUPERPOSITIONS}

Which form can a limit of a convergent (resp. uniformly, strongly, weakly or continuously weakly) sequence of inner superposition operators take between Lebesgue spaces? In this section we give partial answers to this question, which besides being of separate interest for applications also will serve us in the proof of the main results.

4.1. Strong and uniform operator topology. The following result has been proved in 11 for $\Omega=(0,1)$, and can be easily extended for $\Omega \subset \mathbb{R}^{n}$. We present it here with a slight refinement.

Theorem 4.1. Let a sequence of inner superposition operators $T_{g^{\nu}}: L^{p} \rightarrow L^{q}$, converge strongly to an operator $T: L^{p} \rightarrow L^{q}$, where $1 \leq q \leq p<+\infty$. Then $T$ is an inner superposition operator, $T=T_{g}$.

The proof follows immediately from the more general statement below, which extends Lemma 1 of $[10$.

Theorem 4.2. Let a sequence of inner superposition operators $T_{g^{\nu}}: \mathcal{M} \rightarrow \mathcal{M}$ converge strongly to an operator $T: \mathcal{M} \rightarrow \mathcal{M}$. Then $T$ is an inner superposition operator, $T=T_{g}$.

Proof. Setting $u(x)=x$ we see that there is a $g \in \mathcal{M}$ satisfying

$$
\chi_{\mathcal{E}_{\nu}} g^{\nu} \rightarrow \bar{g} \text { in measure, }
$$

while setting $u=1_{\Omega}$, one has the existence of a measurable $\mathcal{E} \subset \Omega$, such that

$$
\chi_{\mathcal{E}_{\nu}} \rightarrow \chi_{\mathcal{E}} \text { in measure. }
$$

Now let $x_{0} \notin \Omega$ and

$$
g(x):=\left\{\begin{array}{cl}
\bar{g}(x), & x \in \mathcal{E} \\
x_{0}, & t \in \Omega \backslash \mathcal{E}
\end{array}\right.
$$

Hence $\mathcal{E}=g^{-1}(\Omega)$.

We would like to show now that $T=T_{g}$. For this purpose, we need first to show that $g: \Omega \rightarrow \mathbb{R}^{n}$ actually generates some operator, i.e.

(i) $\mu_{g}$ is absolutely continuous with respect to Lebesgue measure.

Then, according to Lemma 2 from [9], it will be enough to prove

(ii) $\left|\mathcal{E}_{\nu} \Delta \mathcal{E}\right| \rightarrow 0$;

(iii) $g^{\nu} \rightarrow g$ in measure on $\mathcal{E}$;

(iv) $\mu_{g^{\nu}}$ are uniformly absolutely continuous with respect to Lebesgue measure. 
We proceed in the reverse order. Without changing the operators $T_{g^{\nu}}$, we may assume that $g^{\nu}(x)=x_{0}$ when $x \notin \mathcal{E}_{\nu}$. Now (ii) is immediate, while (iii) follows from

$$
\int_{\mathcal{E}}\left(g^{\nu}(x)-g(x)\right) d x=\int_{\mathcal{E} \backslash \mathcal{E}_{\nu}}\left(g^{\nu}(x)-g(x)\right) d x+\int_{\mathcal{E}}\left(\chi_{\mathcal{E}_{\nu}}(x) g^{\nu}(x)-g(x)\right) d x \rightarrow 0 .
$$

Note that each $\mu_{g^{\nu}}$ is absolutely continuous with respect to Lebesgue measure, and, taking $u:=\chi_{e}$ for measurable $e \subset \Omega$, we have that $\chi_{\mathcal{E}_{\nu}}$ is convergent in measure; hence $\mu_{g^{\nu}}(e)$ converges. This brings into play the classical Vitali-HahnSaks theorem (see Chapter VII of [6]), which provides (iv).

To prove (i), assume the contrary, namely, there exists such $e_{0} \subset \Omega,\left|e_{0}\right|=0$, that $\mu_{g}\left(e_{0}\right)=\beta>0$. Then for any $\varepsilon>0$ there is an open set $\Omega^{\prime}, e_{0} \subset \Omega^{\prime}$, such that $\left|\Omega^{\prime}\right|<\varepsilon$. Hence

$$
\mu_{g}\left(\Omega^{\prime}\right) \geq \beta>0
$$

One observes that there is a subsequence $\left\{\mathcal{E}_{\nu_{\mu}}\left(\Omega^{\prime}\right) \cap \mathcal{E}\right\}$ which tends to $\mathcal{E}\left(\Omega^{\prime}\right)$ in the set-theoretic sense up to a subset of measure zero. Thus

$$
\mu_{g}\left(\Omega^{\prime}\right)=\lim _{\mu}\left|\mathcal{E}_{\nu_{\mu}}\left(\Omega^{\prime}\right) \cap \mathcal{E}\right|,
$$

while (ii) implies $\mu_{g^{\nu}}\left(\Omega^{\prime}\right) \rightarrow 0$ when $\varepsilon \rightarrow 0$ uniformly with respect to $\nu$, which leads to a contradiction.

We remark that the above theorems give a way to construct explicitly the generating function of the limit operator.

Obviously all the previous results hold for uniform convergence too. What actually happens in this case? According to the examples provided in [9], the uniform convergence of inner superposition operators could be expected in rather rare occasions. Unfortunately, this does not mean that the uniform convergence of a nonconstant sequence of inner superpositions acting in Lebesgue spaces is impossible at all, as the following example shows.

Example 4.1. For $\Omega=(0,1)$ let

$$
\begin{aligned}
g^{\nu}(x) & :=\left\{\begin{array}{cl}
2 x, & 0<x \leq 1 / 2, \\
\nu(x-1 / 2), & 1 / 2<x<1,
\end{array}\right. \\
g(x) & :=2 x .
\end{aligned}
$$

It is clear from the direct computation that the operators $T_{g^{\nu}}: L^{p} \rightarrow L^{p}, 1 \leq p<$ $+\infty$, converge uniformly to the operator $T_{g}: L^{p} \rightarrow L^{p}$.

4.2. Weak and weak continuous operator topology. Before considering weak notions of operator convergence, we would like to introduce the following useful convention. Here and everywhere in the sequel when dealing with weak and weak continuous convergence of operators defined on or acting into the space $L^{\infty}(\Omega)$, we understand by weak convergence in this space the $*$-weak one, without changing the notions of operator convergence.

We start with noting that if a sequence of inner superposition operators between Lebesgue spaces converges weakly to an inner superposition operator, then in fact the convergence is strong. This fact, which we present here in the theorem below, was apparently first noted in [11]. 
Theorem 4.3. Let a sequence of inner superposition operators $T_{g^{\nu}}: L^{p} \rightarrow L^{q}$ converge weakly to an operator $T_{g}: L^{p} \rightarrow L^{q}$, while $1 \leq q \leq p<+\infty$. Then the convergence is strong.

Proof. Note that for any measurable set $e \subset \Omega$ one has

$$
\chi_{\mathcal{E}_{\nu}(e)} \rightarrow \chi_{\mathcal{E}(e)} \text { in } L^{q},
$$

but the weak convergence of characteristic function to a characteristic functions implies the strong convergence and, therefore, the statement follows by observing that the density of the linear span of the set

$$
X_{0}=\left\{\chi_{e}: e \subset \Omega \text { is measurable }\right\}
$$

$L^{p}$ and the uniform boundedness of the sequence $T_{g^{\nu}}$.

Remark. It is clear from the proof that the same result holds for $1 \leq q<p=+\infty$. On the contrary, it is false for $q=+\infty$, because a sequence of inner superposition operators acting between $L^{\infty}$ converges strongly, if and only if it is definitely constant.

However, in general weak convergence of inner superposition operators need not be equivalent to the strong convergence, as we show below.

Example 4.2. Let $\Omega=(0,1)$ and the functions $g^{\nu}: \Omega \rightarrow \mathbb{R}, \nu \in \mathbb{N}$, be defined as

$$
g^{\nu}(x)=\nu x-j, \quad j / \nu \leq x<(j+1) / \nu, \quad j=0, \ldots, \nu-1 .
$$

Then the operators $T_{g^{\nu}}: L^{p} \rightarrow L^{p}, 1<p \leq+\infty$, converge weakly continuously to a limit operator $T: L^{p} \rightarrow L^{p}$ given by the formula

$$
(T u)(x):=\int_{\Omega} u(s) d s \cdot 1(x) .
$$

Moreover, if $p=1$, the convergence is only weak. Note that a limit operator is compact, which is impossible for nonzero inner superposition operators [1].

We proceed with the following statement developing the above example.

Proposition 4.1. Let $\Omega \subset D=\prod_{i=1}^{n}\left(a_{i}, b_{i}\right) \subset \mathbb{R}^{n}$ be an open set with locally Lipschitz-continuous boundary. Then for any one-dimensional integral operator $T \in \mathcal{L}\left(L^{p}\right), 1 \leq p \leq+\infty$, of the form

$$
(T u)(x):=1_{\Omega}(x) \frac{1}{|D|} \int_{\Omega} \phi(s) u(s) d s
$$

with $\phi \in C(\bar{\Omega})$,

$$
\phi(x) \geq c>0 \text { and } \frac{1}{|\Omega|} \int_{\Omega} \phi(s) d s=1,
$$

there is a sequence of inner superposition operators in $\mathcal{L}\left(L^{p}\right)$ converging weakly to $T$ so that, if $p>1$, the convergence is also continuous weak.

Proof. Step 1. We need to construct the auxiliary functions $\gamma_{\nu}: D \rightarrow D$, defining its $i$-th component, $i=1, \ldots, n$, by

$$
\gamma_{i}^{\nu}(x):=\nu x_{i}-k\left(b_{i}-a_{i}\right)+b_{i}, \text { if } \frac{x_{i}-a_{i}}{b_{i}-a_{i}} \in\left(\frac{k-1}{\nu}, \frac{k}{\nu}\right), k=1, \ldots, \nu .
$$

We point out that for each $u \in L^{p}(D)$ the function $T_{\gamma^{\nu}} u$ coincides with the restriction to $D$ of $\tilde{u}(\nu(\cdot))$, where $\tilde{u}$ stands for the extension by periodicity of $u$ to 
the whole of $\mathbb{R}^{n}$. Thus, by Theorem 1.5 and the example following its proof in Chapter 2 of [4], $T_{\gamma^{\nu}} \in \mathcal{L}\left(L^{p}(D)\right)$ converge weakly continuously to the operator $S \in \mathcal{L}\left(L^{p}(D)\right)$ defined by

$$
(S u)(x):=1_{D}(x) \frac{1}{|D|} \int_{D} u(s) d s .
$$

Now fix some $x_{0} \notin \Omega$ and define $\bar{g}^{\nu}: D \rightarrow \mathbb{R}^{n}$ by the formula

$$
\bar{g}^{\nu}(x):=\left\{\begin{array}{cc}
\gamma^{\nu}(x), & x \in \Omega \\
x_{0}, & x \notin \Omega
\end{array}\right.
$$

Letting $g^{\nu}: \Omega \rightarrow \mathbb{R}^{n}$ be the restrictions of $\bar{g}^{\nu}$ to $\Omega$, we see that $T_{g^{\nu}} \stackrel{(c w)}{\longrightarrow} \tilde{S}$ in $\mathcal{L}\left(L^{p}\right)$, where

$$
(\tilde{S} u)(x):=1_{\Omega}(x) \frac{1}{|D|} \int_{\Omega} u(s) d s,
$$

because $T_{g^{\nu}} u=\chi_{\Omega} T_{\gamma^{\nu}}$ in $L^{p}(D)$.

Step 2. We use the lemma below to construct a homeomorphism $g: \Omega \rightarrow \Omega$ such that $\frac{d \mu_{g}}{d m}=\phi$. We have then that $T_{g} \in \mathcal{L}\left(L^{p}\right)$ and $T_{g^{\nu}} \circ T_{g} \stackrel{(c w)}{\longrightarrow} \tilde{S} \circ T_{g}=T$ in $\mathcal{L}\left(L^{p}\right)$. It remains to note that $T_{g^{\nu}} \circ T_{g}$ are still inner superpositions.

Lemma 4.1. If $\Omega \subset \mathbb{R}^{n}$ is an open bounded set with locally Lipschitz-continuous boundary and $\phi \in C(\bar{\Omega})$ satisfies

$$
\phi(x) \geq c>0, \quad \frac{1}{|\Omega|} \int_{\Omega} \phi(s) d s=1,
$$

then there is a homeomorphism $g: \Omega \rightarrow \Omega$ satisfying $\frac{d \mu_{g}}{d m}=\phi$.

Proof. According to [5] (see also [16, 15]), there is a homeomorphism $g: \Omega \rightarrow \Omega$ satisfying

in a weak sense, that is,

$$
\operatorname{det} \nabla g^{-1}(x)=\phi(x)
$$

$$
\left|g^{-1}(e)\right|=\int_{e} \phi(s) d s
$$

for all measurable $e \subset \Omega$.

We remark that in principle both Proposition 4.1 and Lemma4.1 could be further refined, for instance, by weakening the regularity requirement on the boundary of $\Omega$. Such a refinement goes, however, beyond the scopes of this paper. We think it also worth emphasizing that the results claimed in both of the above statements are constructive.

Another type of weak limits of inner superpositions is shown in the following example.

Example 4.3. Let $\Omega=(0,1)$ and the functions $g^{\nu}: \Omega \rightarrow \mathbb{R}, \nu \in \mathbb{N}$, be defined as

$$
g^{\nu}(x)=\left\{\begin{array}{ll}
2, & j / \nu \leq x<(2 j+1) / 2 \nu, \\
x, & (2 j+1) / 2 \nu \leq x<j / \nu,
\end{array} \quad j=0, \ldots, 2 \nu .\right.
$$

Then the operators $T_{g^{\nu}}: L^{p} \rightarrow L^{p}, 1 \leq p \leq+\infty$, converge weakly to the limit operator $T: L^{p} \rightarrow L^{p}$ given by the formula

$$
(T u)(x):=\frac{1}{2} u(x)
$$


We develop the idea of the above example in the following statement.

Proposition 4.2. Let $0 \leq a(x) \leq 1$ a.e. in $\Omega$. Then a multiplication operator $T \in \mathcal{L}\left(L^{p}\right), 1 \leq p \leq+\infty$, defined by $(T u)(x):=a(x) u(x)$ is a limit of some weakly convergent sequence of inner superposition operators in $\mathcal{L}\left(L^{p}\right)$.

Proof. Observe that

$$
S:=\left\{\chi_{E}: E \subset \Omega \text { is measurable }\right\} \subset L^{\infty}
$$

is the set of extreme points of

$$
B:=\left\{v \in L^{\infty}(\Omega): 0 \leq v \leq 1 \text { a.e. in } \Omega\right\} .
$$

Thus, given $a \in B$, according to the Krein-Milman theorem there is a sequence $h_{\nu} \stackrel{*}{\rightarrow} a$, where $h_{\nu}$ are convex combinations of characteristic functions. Now, it is easy to note that for each fixed $\nu \in \mathbb{N}$ there is a sequence of characteristic functions $\chi_{E_{\nu, \mu}} \stackrel{*}{\rightarrow} h_{\nu}$ as $\mu \rightarrow \infty$. Recalling that the $*$-weak topology is metrizable over $B$, we conclude that there is a "diagonal" sequence $\chi_{E_{\nu, \mu(\nu)}} \stackrel{*}{\rightarrow} a$. It remains to note that the operators $T_{\nu}: L^{p} \rightarrow L^{p}$ defined by

$$
T_{\nu} u:=\chi_{E_{\nu, \mu(\nu)}} u
$$

are in fact inner superposition operators, while $T_{\nu} \rightarrow T$ in $\mathcal{L}\left(L^{p}\right)$.

Unfortunately, the sequence of operators constructed in the proof of Proposition 4.2 converges only weakly, but not continuously weakly, unless $a \in L^{\infty}$ is a characteristic function. In fact, if $T_{\nu}$ converge continuously weakly to $T$, then taking $u_{\nu}:=\chi_{E_{\nu, \mu(\nu)}}$ we have

$$
T_{\nu} u_{\nu} \rightarrow T a=a^{2} .
$$

On the other hand $T_{\nu} u_{\nu}=u_{\nu} \rightarrow a$, and therefore $a=a^{2}$, i.e. $a \in S$, while the convergence is also strong by Theorem 4.3

At last, we find it suitable to provide the following general necessary conditions for an operator to be a weak limit of inner superposition operators, which sharpen the result announced in [10].

Theorem 4.4. Let a sequence of inner superposition operators $T_{g^{\nu}}: L^{p} \rightarrow L^{q}$ converge weakly to an operator $T: L^{p} \rightarrow L^{q}$, while $1 \leq q \leq p<+\infty$. Then $T$ has the following properties:

(i) $T$ is a positive operator (i.e. maps nonnegative functions in nonnegative ones);

(ii) $\|T\|_{\infty \rightarrow r} \leq|\Omega|^{1 / r}$, where $1 \leq r \leq+\infty$;

(iii) $T \in \mathcal{L}\left(L^{r}, L^{s}\right)$, if either $1 \leq s \leq r<+\infty$ and $r / s \geq p / q$, or $r=+\infty$ and $1 \leq s \leq+\infty$.

Proof. To prove (i), assume the contrary, i.e. the existence of such $u(x) \geq 0$ and $e \in \Sigma,|e|>0$, that $v(x):=(T u)(x)<0$ for $x \in e$. Then

$$
\left|\int_{\Omega}\left((T u)(x)-\left(T_{g^{\nu}} u\right)(x)\right) \chi_{e}(x) d x\right|=\int_{e}|v(x)| d x+\int_{e}\left(T_{g^{\nu}} u\right)(x) d x \geq \int_{e}|v(x)| d x,
$$

which gives the contradiction.

To prove (ii), we show first that $\|T\|_{\infty \rightarrow 1} \leq|\Omega|$. In fact, assume

$$
\|T\|_{\infty \rightarrow 1}=C>|\Omega|
$$


and note that for each $\varepsilon>0$ there exists a $u \in L^{\infty}$ satisfying

$$
\int_{\Omega}|(T u)(x)| d x \geq(C-\varepsilon)\|u\|_{\infty} .
$$

Let $\phi(x):=\operatorname{sign}(T u)(x)$. Then

$$
\begin{gathered}
\left|\int_{\Omega}\left((T u)(x)-\left(T_{g^{\nu}} u\right)(x)\right) \phi(x) d x\right|=\left|\int_{\Omega}\right|(T u)(x)\left|d x-\int_{\Omega}\left(T_{g^{\nu}} u\right)(x) \phi(x) d x\right| \\
\geq(C-\varepsilon-|\Omega|)|| u \|_{\infty}
\end{gathered}
$$

leads to a contradiction.

Now we show $\|T\|_{\infty \rightarrow \infty} \leq 1$. In fact, assuming the contrary, we may assume without loss of generality that there are a $u \in L^{\infty}$ and a measurable $e \subset \Omega,|e|>0$, such that

$$
(T u)(x)>\|u\|_{\infty}, \quad x \in e
$$

Then

$$
|e| \cdot\|u\|_{\infty} \geq \int_{\Omega}\left(T_{g^{\nu}} u\right)(x) \chi_{e}(x) d x \rightarrow \int_{\Omega}(T u)(x) \chi_{e}(x) d x>|e| \cdot\|u\|_{\infty}
$$

gives the contradiction.

The rest of the statement (ii) follows from the interpolation theorem for positive linear operators (Theorem 2.5 in [14]).

Now we concentrate on the proof of (iii). The case $r=+\infty$ is in fact included in (ii). As for the case $r<+\infty$, it is enough to prove that $T \in \mathcal{L}\left(L^{r}, L^{s}\right)$ when $r / s=p / q$, since the rest follows from the obvious general properties of linear operators acting in Lebesgue spaces ( $\operatorname{see} \S 1.6$ of [14]). At last, the case $r / s=p / q$ follows immediately from the cited interpolation theorem (because $T \in \mathcal{L}\left(L^{r}, L^{s}\right)$ for $r=s=+\infty$ by (ii) and for $r=p, s=q$ ).

\section{LiNEAR COMBINATIONS OF INNER SUPERPOSITIONS AND THEIR LIMITS}

In this section we deal with limits of linear combinations of inner superposition operators, adopting the notation

$$
\Theta_{p, q}:=\operatorname{span}\left\{T_{g} \in \mathcal{L}\left(L^{p}, L^{q}\right)\right\}, \quad \Theta_{p}:=\Theta_{p, p}
$$

We will further be interested also in a particularly "nice" subset $\Theta_{p, q}^{\prime}$ of $\Theta_{p, q}$, namely, by a span of inner superposition operators generated by piecewise homeomorphisms. As above, let $\Theta_{p}^{\prime}:=\Theta_{p, p}^{\prime}$.

From now on we will always assume $\Omega \subset \mathbb{R}^{n}$ to be an open bounded subset with locally Lipschitz-continuous boundary. We start with the following result.

Proposition 5.1. Let $\phi \in L^{\infty}$. There is a sequence of linear combinations of inner superpositions in $\mathcal{L}\left(L^{p}\right), 1<p<+\infty$, which converges continuously weakly to the integral operator $T: L^{p} \rightarrow L^{p}$ defined by

$$
(T u)(x):=1_{\Omega}(x) \int_{\Omega} \phi(s) u(s) d s .
$$

Proof. Step 1. Write $\phi=\phi^{+}-\phi^{-}$, where $\phi^{+}$and $\phi^{-}$are the positive and negative parts of $\phi$ respectively, i.e. $\phi^{+}(x):=\max (\phi(x), 0)$ and $\phi^{-}:=(-\phi)^{+}$. We note that 
it is enough to find the sequences $\left\{T_{\nu}^{+}\right\}$and $\left\{T_{\nu}^{-}\right\}$in $\Theta_{p}$, converging continuously weakly to $T^{+}$and $T^{-}$respectively, where

$$
\left(T^{+} u\right)(x):=1_{\Omega}(x) \int_{\Omega} \phi^{+}(s) u(s) d s, \quad\left(T^{-} u\right)(x):=1_{\Omega}(x) \int_{\Omega} \phi^{-}(s) u(s) d s .
$$

The desired sequence will then be $\left\{T_{\nu}^{+}-T_{\nu}^{-}\right\}$.

Step 2. According to the previous step, we may assume without loss of generality that $\phi \geq 0$. Now we approximate $T$ uniformly by one-dimensional integral operators with "regular" kernels. For all $\nu \in \mathbb{N}$ let $\bar{\phi}_{\nu} \in C(\bar{\Omega})$ be such that

$$
\left\|\bar{\phi}_{\nu}-\phi\right\|_{p}<1 / \nu
$$

and set $\phi_{\nu}(x):=\max \left(\bar{\phi}_{\nu}, 1 / \nu\right)$. Then $\phi_{\nu} \rightarrow \phi$ in $L^{p^{\prime}}, \phi_{\nu} \in C(\bar{\Omega})$ and $\phi_{\nu} \geq 1 / \nu$ in $\Omega$. Thus the operators $S_{\nu}: L^{p} \rightarrow L^{p}$ defined by

$$
\left(S_{\nu} u\right)(x):=1_{\Omega}(x) \int_{\Omega} \phi_{\nu}(s) u(s) d s
$$

converge uniformly to $T$.

Step 3. According to Proposition 4.1, for each fixed $\nu \in \mathbb{N}$ one can construct a sequence of inner superposition operators $T_{g^{\nu, \mu}}: L^{p} \rightarrow L^{p}$, such that

$$
\lambda_{\nu} T_{g^{\nu, \mu}} \stackrel{(c w)}{\longrightarrow} S_{\nu} \text { as } \mu \rightarrow \infty
$$

where $\lambda_{\nu}:=\left\|\phi_{\nu}\right\|_{1} \cdot|D| /|\Omega|$ and $D \subset \mathbb{R}^{n}$ is some cube containing $\Omega$. Since $\phi \in L^{\infty}$, we may assume that the sequence $\left\{\bar{\phi}_{\nu}\right\}$, and hence $\left\{\phi_{\nu}\right\}$, is uniformly bounded in $C(\bar{\Omega})$. Thus by Lemma 4.1 the norms of all the operators $\left\{\lambda_{\nu} T_{g^{\nu, \mu}}\right\}$ are uniformly bounded and by Lemma 3.2, there is a "diagonal" sequence

$$
\lambda_{\nu} T_{g^{\nu, \mu(\nu)}} \stackrel{(c w)}{\longrightarrow} T
$$

which concludes the proof.

Let us remark that the operators of the approximating sequence constructed in the proof of the above proposition are generated by piecewise homeomorphisms.

We prove now the first principal result of this paper.

Theorem 5.1. Let $1<q \leq p<+\infty$. Any operator in $\mathcal{L}\left(L^{p}, L^{q}\right)$ is a weak continuous limit of some sequence of operators, which can be obtained as sequential weak continuous limits of linear combinations of inner superposition operators generated by piecewise homeomorphisms. In particular, the linear span of such "nice" inner superposition operators $\Theta_{p, q}^{\prime}$ is dense in continuous weak topology of $\mathcal{L}\left(L^{p}, L^{q}\right)$.

Proof. Step 1. We recall the following result of "folklore" character.

Lemma 5.1. Any operator from $\mathcal{L}\left(L^{p}, L^{q}\right), 1 \leq p \leq+\infty, 1 \leq q<+\infty$, is a limit of some sequence of finite rank operators converging strongly and, if $q>1$, also continuously weakly.

Proof of Lemma 5.1. To prove the strong approximation result, it is enough to show that there is a sequence of finite rank operators $\left\{T_{\nu}\right\} \subset \mathcal{L}\left(L^{q}\right)$ converging strongly to the identity. In fact, in this case any operator $A \in \mathcal{L}\left(L^{p}, L^{q}\right)$ is approximated by a strongly converging sequence of operators $\left\{T_{\nu} \circ A\right\}$ of finite rank.

Let $\left\{u_{\nu}\right\}$ stand for the Haar system in $L^{q}$ (see [6] ). Then defining

$$
T_{\nu} u:=\sum_{i=1}^{\nu}\left\langle u, u_{i}^{\prime}\right\rangle u_{i}
$$


where $\left\{u_{i}^{\prime}\right\}$ is a system of functionals biorthogonal to $\left\{u_{i}\right\}$, which in fact is constituted by the same functions, we conclude this part of the proof referring to the fact that $\left\{u_{\nu}\right\}$ is a Schauder basis in $L^{q}, 1 \leq q<+\infty$.

To prove the continuous weak approximation result, it remains to observe that, if $1<q<+\infty$, then $T_{\nu}^{\prime} \rightarrow I d$ strongly, and hence, for any operator $A \in \mathcal{L}\left(L^{p}, L^{q}\right)$, the sequence $\left\{T_{\nu} \circ A\right\}$ converges to $A$ also continuously weakly by Lemma 3.1 . because the sequence of adjoints $\left\{A^{\prime} \circ T_{\nu}^{\prime}\right\}$ converge strongly to $A^{\prime}$.

We note now that every finite rank operator in $\mathcal{L}\left(L^{p}, L^{q}\right)$ can be approximated uniformly by finite rank operators of a particular form

$$
\left(T_{\nu} u\right)(x):=\sum_{i=1}^{\nu} a_{i}(x) \int_{\Omega} u(s) \phi_{i}(s) d s
$$

with all $\phi_{i} \in L^{\infty}$. We will show that all such operators can be approximated in continuous weak sense by means of operators from $\Theta_{p, q}^{\prime}$. The desired assertion will follow then from Lemma 3.2 since the condition $(*)$ of this lemma is automatically satisfied in case of uniform convergence.

Step 2. Let $T: L^{p} \rightarrow L^{q}$ be a finite rank operator,

$$
(T u)(x):=a(x) \int_{\Omega} \phi(s) u(s) d s,
$$

where $\phi \in L^{\infty}, a \in L^{q}$. Let $T_{\nu}: L^{p} \rightarrow L^{q}$ be defined by

$$
\left(T_{\nu} u\right)(x):=a_{\nu}(x) \int_{\Omega} \phi(s) u(s) d s,
$$

where $a_{\nu} \in C(\bar{\Omega})$ and $\left\|a_{\nu}-a\right\|_{q}<1 / \nu$. Hence, $T_{\nu} \rightarrow T$ uniformly in $\mathcal{L}\left(L^{p}, L^{q}\right)$. If we show now that for each $\nu \in \mathbb{N}$ there is a sequence $\left\{T_{\nu, \mu}\right\} \subset \Theta_{p, q}^{\prime}$ such that $T_{\nu, \mu} \stackrel{(c w)}{\longrightarrow} T_{\nu}$ as $\mu \rightarrow \infty$, then the proof is concluded by extracting a diagonal sequence according to Lemma 3.2 .

Step 3. It remains thus to show that the rank one operator $T$ defined on Step 2 may be approximated in the continuous weak sense by a sequence from $\Theta_{p, q}^{\prime}$, if $a \in C(\bar{\Omega})$. Since we may find a sequence

$$
\left\{\hat{a}_{\nu}\right\} \subset X_{0}:=\operatorname{span}\left\{\chi_{E}: E \subset \Omega \text { is measurable }\right\},
$$

such that $\hat{a}_{\nu} \rightarrow a$ in $L^{\infty}$, there is a sequence of operators $\hat{T}_{\nu}: L^{p} \rightarrow L^{q}$ of the form

$$
\left(\hat{T}_{\nu} u\right)(x):=\hat{a}_{\nu}(x) u(x),
$$

such that $\hat{T}_{\nu} \rightarrow \hat{T}$ uniformly in $\mathcal{L}\left(L^{p}, L^{q}\right)$, where

$$
(\hat{T} u)(x):=a(x) u(x) .
$$

Note that $\left\{\hat{T}_{\nu}\right\} \subset \Theta_{p, q}^{\prime}$. According to Proposition 5.1 there is a sequence $\left\{\bar{T}_{\nu}\right\} \subset \Theta_{p}^{\prime}$ converging continuously weakly to $\bar{T}$ in $\mathcal{L}\left(L^{p}\right)$, where

$$
(\bar{T} u)(x):=1_{\Omega}(x) \int_{\Omega} \phi(s) u(s) d s .
$$

We conclude by observing that

$$
\hat{T}_{\nu} \circ \bar{T} \stackrel{(c w)}{\longrightarrow} \hat{T} \circ \bar{T}=T
$$

while $\hat{T}_{\nu} \circ \bar{T} \in \Theta_{p, q}^{\prime}$. 
Corollary 5.1. The linear span $\Theta_{p, q}^{\prime}$ of the set of inner superposition operators generated by piecewise homeomorphisms is dense in the strong operator topology of $\mathcal{L}\left(L^{p}, L^{q}\right)$, where $1<q \leq p<+\infty$.

Proof. From the above Theorem 5.1 it follows that $\Theta_{p, q}^{\prime}$ is dense in the weak operator topology of $\mathcal{L}\left(L^{p}, L^{q}\right)$. It is enough now to refer to Theorem VI.1.4 of [12], according to which the duals of the spaces $\mathcal{L}\left(L^{p}, L^{q}\right)$ equipped with strong and weak operator topology respectively coincide.

It is important to emphasize that the strong topology in $\mathcal{L}\left(L^{p}, L^{q}\right)$ is not metrizable, and hence the result provided by the above corollary in principle does not allow us to assert that any linear operator between Lebesgue spaces is a strong limit of a sequence of linear combinations of inner superpositions. However, we will be able to show now that such a stronger sequential result still takes place. For this purpose we employ the following lemma, which is a by-product of Theorem 3 of $[3]$.

Lemma 5.2. There is a sequence of linear combinations of inner superpositions in $\mathcal{L}\left(L^{p}\right), 1<p<+\infty$, which converges uniformly to an integral operator $T: L^{p} \rightarrow L^{p}$ defined by

$$
(T u)(x):=1_{\Omega}(x) \frac{1}{|\Omega|} \int_{\Omega} u(s) d s .
$$

To prove the lemma, it is enough to follow the lines of the proof of the mentioned Theorem 3 of [3]. Here we report only the main idea of the construction.

Idea of the proof of Lemma 5.2. In [3] it has been proved, using the theorem on isomorphism for measure spaces [13, that in a standard probability space $(\Omega, \Sigma, \mu)$ for each fixed $\nu \in \mathbb{N}$ there exists a finite collection of measure preserving maps

$$
g_{i}^{\nu}: \Omega \rightarrow \Omega, \quad i=1, \ldots, \nu,
$$

such that a family of $\sigma$-subalgebrae $\left\{\left(g_{i}^{\nu}\right)^{-1}(\Sigma)\right\}_{i=1}^{\nu}$ is independent in totality. It may be shown now, with the help of an interpolation theorem for positive linear operators [14], that the sequence $\left\{T_{\nu}\right\} \subset \Theta_{p}$, defined by

$$
\left(T_{\nu} u\right)(x):=\frac{1}{\nu} \sum_{i=1}^{\nu}\left(T_{g_{i}^{\nu}} u\right)(x)
$$

converges uniformly to $T$.

It seems important to point out that the maps $g_{i}^{\nu}$ with special properties, which constitute the heart of the proof, are defined in a rather nonconsructive way: in practice, their existence is provided by the theorem on isomorphism of measure spaces [13]. Moreover, as it has been shown in [3, these maps should necessarily possess some rather pathological properties, e.g. they can be injective only on a set of zero measure, while the preimage of almost any point is nondenumerable. In fact, in [3] such maps are called "extremely noninvertible" or "anti-injective".

Now, using Lemma 5.2 and following the lines of the proof of Proposition 5.1 we may approximate uniformly by linear combinations of inner superpositions any integral operator $T: L^{p} \rightarrow L^{p}$ of more general form

$$
(T u)(x):=1_{\Omega}(x) \int_{\Omega} \phi(s) u(s) d s, \quad \phi \in L^{\infty} .
$$


It remains at last to reiterate the arguments of the proof of Theorem 5.1 to get the following statement, which is the second principal result of this paper, and is even slightly stronger than just a strong sequential density result.

Theorem 5.2. Any operator in $\mathcal{L}\left(L^{p}, L^{q}\right), 1<q \leq p<+\infty$, is a limit of some sequence of linear combinations of inner superposition operators, converging both strongly and continuously weakly.

Finally we remark again that the generating functions involved are no more piecewise homeomorphisms, but have the rather pathological character of "extreme noninvertibility".

\section{ACKNOWLEDGEMENTS}

The authors are most grateful to Professor M. E. Drakhlin (Ariel Research Inst. of Mathematics, Israel) for numerous stimulating discussions during the work on this paper.

\section{REFERENCES}

[1] N. V. Azbelev, V. P. Maksimov, and L.F. Rakhmatullina. Introduction to the Theory of Functional Differential Equations. "Nauka", Moscow, 1991. in Russian. MR 92j:34123. English transl. of the 1st part: Introduction to the Theory of Linear Function Differential Equations. World Federation Publishers, 1996. MR 97m:34002

[2] G. Buttazzo, M.E. Drakhlin, L. Freddi, and E. Stepanov. Homogenization of optimal control problems for functional differential equations with deviating argument. J. Optim. Theory and Appl., 93(1):103-119, 1997. MR 98d:49015

[3] A.V. Chistyakov. A pathological counterexample for hypothesis of non-Fredholm property in algebras of weighted shift operators. Izvestiya VUZ. Matematika, 39(10):76-86, 1995. in Russian. English transl.: Russian Mathematics, 39(10):73-83, 1995.

[4] B. Dacorogna. Direct Methods in the Calculus of Variations, volume 78 of Applied Mathematical Sciences. Springer Verlag, Berlin, Heidelberg, 1989. MR 90e:49001

[5] B. Dacorogna and J. Moser. On a partial differential equation involving the Jacobian determinant. Ann. Inst. H. Poincaré. Analyse Nonlinéaire, 7(1):1-26, 1990. MR 91i:58148

[6] J. Diestel. Sequences and Series in Banach Spaces, volume 92 of Graduate Texts in Mathematics. Springer-Verlag, Berlin, 1984. MR 85i:46020

[7] M. E. Drakhlin and E. Stepanov. $\Gamma$-convergence for a class of functionals with deviating argument. J. Convex Analysis, 4(1):69-89, 1997. MR 98j:49022

[8] M.E. Drakhlin. An inner superposition operator in spaces of summable functions. Izvestiya VUZ. Matematika, 30(5):18-24, 1986. in Russian. MR 88b:47039.

[9] M.E. Drakhlin. On convergence of sequences of internal superposition operators. Functional Diff. Equations. Israel Seminar, 1:83-94, 1993. MR 95g:47047

[10] M.E. Drakhlin and E. Stepanov. Convergence of composition operators and optimal control problems. Proc. of 2nd World Congress on Nonlinear Analysis, 30:505-512, 1997. MR 99k:47064

[11] M.E. Drakhlin and E. Stepanov. Weak convergence of inner superposition operators. Proc. Amer. Math. Soc., 126(1):173-179, 1998. MR 98c:47038

[12] N. Dunford and J.T. Schwartz. Linear Operators, vol. 1, General Theory. Interscience Publ. Ltd. N.Y., 1958. MR 22:8302

[13] R.P. Halmos. Measure Theory, volume 18 of Graduate Texts in Mathematics. Springer-Verlag, Berlin, 1974.

[14] M. A. Krasnoselskiǐ, P. P. Zabrě̌ko, E.I. Pustyl'nik, and P.E. Sobolevskiǐ. Integral Operators in the Spaces of Summable Functions. "Nauka", Moscow, 1966. in Russian. English transl.: Noordhoff International Publishing, Leyden, 1975. 
[15] J. Moser. On the volume element on a manifold. Trans. Amer. Math. Soc., 120:286-294, 1965. MR 32:409

[16] T. Rivière and D. Ye. Un resolution de l'equation a forme volume prescrit. Comptes Rendus Acad. Sci. Paris. Ser. I.-Math., 319(1):25-28, 1994. in French. MR 95f:35055.

Dipartimento di Matematica, Universitá di Pisa, via Buonarrotti 2, 56127 Pisa, Italy

Scuola Normale Superiore, Piazza dei Cavalieri 7, 56126 Pisa, Italy

E-mail address: depascal@dm.unipi.it

Computer Technology Department, St. Petersburg Inst. of Fine Mechanics and Optics, 14 Sablinskaya ul., 197101 St. Petersburg, Russia 